\title{
Does resveratrol act independently of SIRT1 to affect genes relevant to ageing?
}

\author{
S. M. Escolme, L. A. Wakeling, F. Alatawi, R. Valentine and D. Ford \\ Institute for Cell and Molecular Biosciences, Newcastle University, Newcastle Upon Tyne, NE2 4HH, UK
}

Some effects of the polyphenol resveratrol mimic responses to dietary restriction. Dietary resveratrol may thus have the potential to prolong lifespan and reduce the risk of age related diseases, such as cardiovascular disease, diabetes and cancer. Actions of the histone deacetylase Sirt1 are one of several pathways believed to play a role in responses to dietary restriction. Controversy remains over whether or not resveratrol is an activator of SIRT1 and thus over if SIRT1 is the conduit for the beneficial actions of resveratrol.

Previous work in human intestinal Caco-2 cells, based on DNA microarray analysis, indicated that resveratrol and SIRT1 affect distinct sets of genes, although genes affected by dietary restriction were significantly over-represented in both groups. The aim was to investigate if resveratrol and SIRT1 act independently to affect the expression of different genes with functions relevant to ageing.

Clusterin and WWP1 were selected from the group of genes previously found to be affected by resveratrol and dietary restriction. Clusterin has been detected at high levels in Alzheimer's disease and modulates plaque solubility. WWP1 is a ubiquitin ligase that increased lifespan when overexpressed in C. elegans and when mutated led to abolition of the longevity response to dietary restriction. $L A M P 2$, a key player in chaperone-mediated autophagy, which modulates cellular ageing, was selected from the group of genes that were affected by SIRT1 and dietary restriction. RT-qPCR was used to measure mRNA levels of all three genes and of SIRT1in human intestinal Caco- 2 cells after treatment with resveratrol or reduction of SIRT1 expression using siRNA.

Results are shown in Figure 1. SIRT1 knockdown increased Clusterin and WWP1 mRNA and decreased LAMP2 mRNA. Resveratrol decreased WWP1 mRNA and increased SIRT1 mRNA. Resveratrol also increased transcription from a SIRT1 promoter-reporter construct.
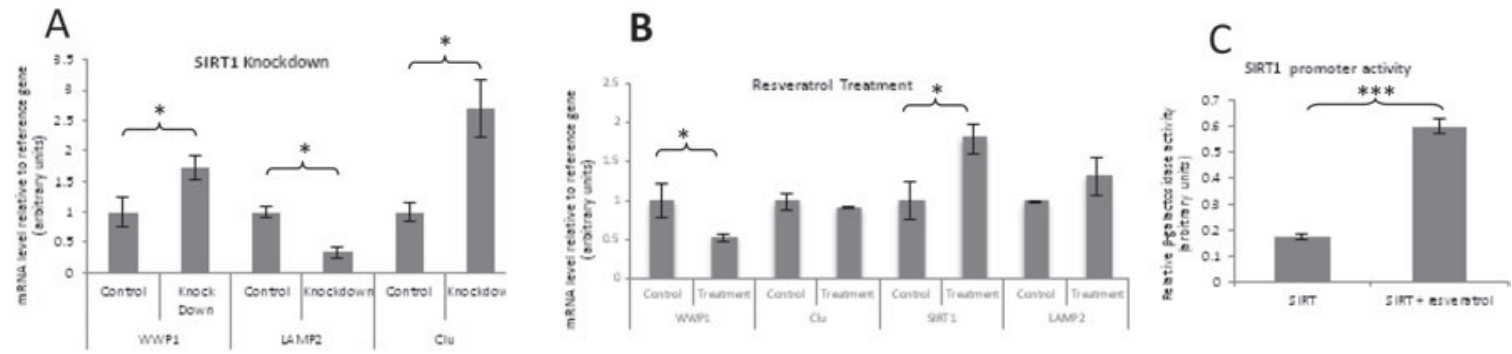

Fig. 1. A. mRNA levels of WWP1, Clusterin $(C l u)$ and $L A M P 2$ relative to GAPDH in response to SIRT1 knockdown in Caco-2 cells B. mRNA levels of WWP1, Clusterin, $L A M P 2$ and SIRT1 relative to GAPDH in response to treatment of Caco- 2 cells over $24 \mathrm{~h}$ with $10 \mu \mathrm{M}$ resveratrol. C. SIRT1 promoter-reporter assay to investigate the effect of resveratrol on the transcription of the SIRTI gene. Data are mean, SEM $(n=3)$. $* P<0.05 ; * * * P<0.001$ by Student's $t$-test.

The observed response of the WWP1 gene to SIRT1 knockdown and to resveratrol is consistent with resveratrol acting via SIRT1. The observed effects of resveratrol on SIRT1 expression indicate that resveratrol can affect the expression of genes through increasing SIRTI transcription. Thus effects of resveratrol on gene expression are not all independent of actions on SIRT1. 